\title{
Multiple Positive Solutions of Two-Point Boundary Value Problems for Systems of Nonlinear Third-Order Differential Equations ${ }^{1}$
}

\author{
Zhongmin Sun, Suoquan Ren, Zhenlin Wu, Huabin Zhang \\ Weifang Education College, Qingzhou, Shandong Province, China
}

\begin{abstract}
In this paper, we study the two-point boundary value problems for systems of nonlinear third-order dierential equations .Under some conditions, we show the existence and multiplicity of positive solutions of the above problem by applying the fixed point theorems in cones.
\end{abstract}

Index Terms: Boundary value problems; Positive solutions; Green's functions; cones

(C) 2012 Published by MECS Publisher. Selection and/or peer review under responsibility of the Research Association of Modern Education and Computer Science.

\section{Introduction}

Recently, there is much attention paid to the existence of positive solutions for third-order nonlinear boundary value problems(see [1-5] and references cited therein)

In [6] Moustafa El-shahed discussed the existences of positive solutions for the following boundary value problem:

$$
\left\{\begin{array}{l}
u^{\prime \prime \prime}(t)+\lambda a(t) f(u(t))=0, \\
u(0)=u^{\prime}(0)=0, \quad \alpha u^{\prime}(1)+\beta u^{\prime \prime}(1)=0 .
\end{array}\right.
$$

By using a Krasnosel'skii'_xed-point theorem, the existence of solutions of the problem (1.1)is obtained in the case when, either $\mathrm{f}$ is superlinear, or $\mathrm{f}$ is sublinear. Zhi-Lin Yang [7] etal.considered the existence and multiplicity of positive solutions for boundary value problems

\footnotetext{
${ }^{1}$ Supported by National Natural Science Foundation of P.R.China(No:10671012)

* Corresponding author.

E-mail address: szm6970@ sina.com
} 


$$
\left\{\begin{array}{c}
-u^{\prime \prime}=f(t, v) \\
-v^{\prime \prime}=g(t, u) \\
u(0)=u(1) \\
v(0)=v(1)=0
\end{array}\right.
$$

Motived by the works of [6] and [7], this paper is concerned with the existence of positivesolution for boundary value problem

$$
\begin{cases}-u^{\prime \prime \prime}=f(t, v), & \\ -v^{\prime \prime \prime}=g(t, u), & \\ u(0)=u^{\prime}(0)=0, & \alpha u^{\prime}(1)+\beta u^{\prime \prime}(1)=0 \\ v(0)=v^{\prime}(0)=0, & \alpha v^{\prime}(1)+\beta v^{\prime \prime}(1)=0 .\end{cases}
$$

where $\left.f, g \in C[0,1] \times R^{+}, R^{+}\right), \alpha \geq 0, \beta \geq 0$.

The arguments for establishing the existence of solutions of BVP (1.3) involve properties of Green's functions that play a key role in the de_nition of cones. A_xed point theorem due to krasnosel'skii [11] is applied to yield the existence of posi-tive solutions of BVP (1.3). Another fixed point theorem about multiplicity is applied to obtain the multiplicity of positive sol-utions of BVP (1.3).

This paper is organized as follows. In the next section, we present some notation and preliminaries. The main results, existence and multiplicity of positive solutions of BVP (1.3)

are given in section 3. Some examples to illustrate our main results appear also in section 3 .

\section{Preliminaries}

Obviously $(u, v) \in C^{3}[0,1] \times C^{3}[0,1]$ is the solution of BVP (1.3) if and only if it satisfies the system of integral equations

$$
\left\{\begin{array}{l}
u(t)=\int_{0}^{1} G(t, s) f(s, v(s)) d s, \\
v(t)=\int_{0}^{1} G(t, s) g(s, u(s)) d s,
\end{array}\right.
$$

where $\mathrm{G}(\mathrm{t} ; \mathrm{s})$ is the Green's function de_ned by

$$
G(t, s)=\left\{\begin{array}{cc}
\frac{\alpha t^{2}(1-s)}{2(\alpha+\beta)}+\frac{\beta t}{2(\alpha+\beta)}, & 0 \leq t \leq s \leq 1 \\
\frac{\alpha t^{2}(1-s)}{2(\alpha+\beta)}+\frac{\beta t^{2}}{2(\alpha+\beta)}-\frac{(t-s)^{2}}{2}, & 0 \leq s \leq t \leq 1
\end{array}\right.
$$

Integral equations (2.1) can be transferred to the nonlinear int-egral equation 


$$
u(t)=\int_{0}^{1} G(t, s) f\left(\left(s, \int_{0}^{1} G(s, \tau) g(\tau, u(\tau)) d \tau\right)\right) d s .
$$

Lemma 1. The Green's function $\mathrm{G}(\mathrm{t}, \mathrm{s})$ satisfies.

(i) $\quad G(t, s) \geq q(t) G(1, s)$, for $0 \leq t, s \leq 1$,

(i) $\quad G(t, s) \geq 0$, and $G(t, s) \leq G(1, s), \quad 0 \leq t, s \leq 1 ; \quad$ where $\quad q(t)=\frac{\beta t^{2}}{\alpha+\beta}$

Proof. If $t \leq s$, then

$$
\begin{aligned}
\frac{G(t, s)}{G(1, s)} & =\frac{\frac{\alpha}{\alpha+\beta} \frac{t^{2}(1-s)}{2}+\frac{\beta}{\alpha+\beta} \frac{t^{2}}{2}}{\frac{\alpha}{\alpha+\beta} \frac{(1-s)}{2}+\frac{\beta}{\alpha+\beta} \frac{1}{2}}=\frac{\frac{\varepsilon}{\alpha+\beta} t^{2}(1-s)+\frac{\beta}{\alpha+\beta} t^{2}}{1-\frac{\alpha s}{\alpha+\beta}} \\
& \geq \frac{\alpha}{\alpha+\beta} t^{2}(1-s)+\frac{\beta}{\alpha+\beta} t^{2} \\
& \geq \frac{\beta}{\alpha+\beta} t^{2}
\end{aligned}
$$

If $\mathrm{t} \geq \mathrm{s}$, then

$$
\begin{aligned}
G(t, s)-t^{2} G & (1, s) \\
= & \frac{1}{2}\left[\frac{\alpha t^{2}(1-s)}{\alpha+\beta}+\frac{\beta t^{2}}{\alpha+\beta}-(t-s)^{2}-t^{2}\left(\frac{\alpha(1-s)}{\alpha+\beta}+\frac{\beta}{\alpha+\beta}+(1-s)^{2}\right)\right] \\
& =\frac{1}{2}\left(t^{2} s^{2}-2 t^{2} s-s^{2}+2 t s\right) \\
& =\frac{1}{2}(s(1-t)[2 t-s(1+t)]) \geq 0
\end{aligned}
$$

so that

$$
\frac{G(t, s)}{G(1, s)} \geq t^{2} \geq \frac{\beta}{\alpha+\beta} t^{2}
$$

The result of (ii) is obvious. The proof is complete.

Let $\mathrm{E}=\mathrm{C}[0,1]$. For $u \in E$, define $\|u\|=\max _{0 \leq t \leq 1}|u(t)|$.

Then $(E,\|\bullet\|)$ is a Banach space. Denote

$$
P=\{u \in E \mid u(t) \geq 0, u(t) \geq q(t)\|u\|, t \in[0,1]\}
$$

It is obvious that $\mathrm{P}$ is a positive cone in $\mathrm{E}$. Define 
Lemma 2. If the operator $\mathrm{A}$ is defined in (2.3), the $\mathrm{A}: \mathrm{P} \rightarrow \mathrm{P}$ is completely continuous.

Proof. From the continuity of $\mathrm{f}$ and $\mathrm{g}$, we know $A u \in E$ for each $u \in P$. It follows from Lemma 1 that for $u \in P$

$$
\begin{aligned}
A u(t) & =\int_{0}^{1} G(t, s) f\left(s, \int_{0}^{1} G(s, \tau) g(\tau, u(\tau)) d \tau\right) d s \\
& =q(t) \int_{0}^{1} G(t, s) f\left(s, \int_{0}^{1} G(s, \tau) g(\tau, u(\tau)) d \tau\right) d s \\
& =q(t)\|A u\|, \quad u \in P .
\end{aligned}
$$

Therefore $A: P \rightarrow P$. Since $G(t, s), f(t, v)$ and $g(t, u)$ are continuous, it is easy to show that $A: P \rightarrow P$ is completely continuous. The proof is complete.

Lemma 3. [11] Let $(\mathrm{E},\|\cdot\|)$ be a Banach space, and $\mathrm{P} \subset \mathrm{E}$ a cone in $\mathrm{E}$. Assume that $\Omega_{1}$ and $\Omega_{2}$ are open subset of E such that $0 \in \Omega_{1}, \bar{\Omega}_{1} \subset \Omega_{2}$. If

$$
A: P \cap\left(\bar{\Omega}_{2} \backslash \Omega_{1}\right) \rightarrow P
$$

is a completely continuous operator such that either

$$
\begin{aligned}
& \text { (i) }\|A u\| \leq\|u\|, u \in P \cap \partial \Omega_{1} \text {, and }\|A u\| \geq\|u\|, u \in P \cap \partial \Omega_{2} \text {,or } \\
& \text { (ii) }\|A u\| \geq\|u\|, u \in P \cap \partial \Omega_{1} \text {, and }\|A u\| \leq\|u\|, u \in P \cap \partial \Omega_{2} .
\end{aligned}
$$

then $\mathrm{A}$ has a fixed point in $P \cap\left(\bar{\Omega}_{2} \backslash \Omega_{1}\right)$

Lemma 4. [9,10] Let (E, $\|\cdot\|$ ) be a Banach space, and $P \subset E$ a cone in E. Assume that $\Omega_{1}, \Omega_{2}$ and $\Omega_{3}$ are open subset of E such that $0 \in \Omega_{1}, \bar{\Omega}_{1} \subset \Omega_{2}, \bar{\Omega}_{2} \subset \Omega_{3}$. If

$$
A: P \cap\left(\bar{\Omega}_{3} \backslash \Omega_{1}\right) \rightarrow P
$$

is a completely continuous operator such that

$$
\begin{array}{ll}
\|A u\| \geq\|u\|, & u \in P \cap \partial \Omega_{1} ; \\
\|A u\| \leq\|u\|, & A u \neq u_{1} \quad \forall u \in P \bigcap \partial \Omega_{2} ; \\
\|A u\| \geq\|u\|, \quad & \forall u \in P \bigcap \partial \Omega_{3}, \text { and }\|A u\| \leq\|u\|, u \in P \cap \partial \Omega_{2} .
\end{array}
$$

then A has at least two fixed point $x^{*}, x^{* *}$ in $P \cap\left(\bar{\Omega}_{3} \backslash \Omega_{1}\right)$ and furthermore $x^{*} \in P \cap\left(\bar{\Omega}_{2} \backslash \Omega_{1}\right), x^{* *} \in P \cap\left(\bar{\Omega}_{3} \backslash \bar{\Omega}_{2}\right)$.

\section{Main results}

First we give the following assumptions: 
$\left(A_{1}\right) \lim _{u \rightarrow 0^{+}} \sup _{t \in[0,1]} \frac{f(t, u)}{u}=0, \quad \lim _{u \rightarrow 0^{+}} \sup _{t \in[0,1]} \frac{g(t, u)}{u}=0$

$\left(A_{2}\right) \lim _{u \rightarrow \infty} \inf _{t \in[0,1]} \frac{f(t, u)}{u}=\infty, \quad \lim _{u \rightarrow \infty} \inf _{t \in[0,1]} \frac{g(t, u)}{u}=\infty$

$\left(A_{3}\right) \lim _{u \rightarrow 0^{+}} \inf _{t \in[0,1]} \frac{f(t, u)}{u}=\infty, \quad \lim _{u \rightarrow 0^{+}} \inf _{t \in[0,1]} \frac{g(t, u)}{u}=\infty$

$\left(A_{4}\right) \lim _{u \rightarrow \infty} \sup _{t \in[0,1]} \frac{f(t, u)}{u}=0, \quad \lim _{u \rightarrow \infty} \sup _{t \in[0,1]} \frac{g(t, u)}{u}=0$

$\left(A_{5}\right) f(t, u), g(t, u)$

are increasing functions with respect to $\mathrm{u}$ and there is a number $\mathrm{N}>0$ such that

$$
f\left(t, \frac{1}{2} \int_{0}^{1} g(s, N) d s\right)<2 N \quad \forall t \in[0,1], s \in[0,1]
$$

Theorem 1 If $\left(A_{1}\right)$ and $\left(A_{2}\right)$ are satisfied, then BVP (1.3) has at least one positive solution

$$
(u, v) \in C^{3}\left([0,1], R^{+}\right) \times C^{3}\left([0,1], R^{+}\right)
$$

Satisfying $u(t)>0, v(t)>0$.

Proof. From $\left(A_{1}\right)$ there is a number $H_{1} \in(0,1)$, such that for each $(u, v) \in[0,1] \times\left(0, H_{1}\right)$, one has

$$
f(t, u) \leq \eta u \quad g(t, u) \leq \eta u,
$$

where $\eta>0$.

satisfies $\eta \int_{0}^{1} G(1, s) d s \leq 1$

For every $u \in P$ and $\|u\|=\frac{H_{1}}{2}$,

note that

$$
\int_{0}^{1} G(s, \tau) g(\tau, u(\tau)) d \tau \leq \eta \int_{0}^{1} G(1, \tau) u(\tau) d \tau \leq\|u\|=\frac{H_{1}}{2}<H_{1}
$$


thus

$$
\begin{aligned}
A u(t) & \leq \int_{0}^{1} G(1, s) f\left(s, \int_{0}^{1} G(1, \tau) g(\tau, u(\tau)) d \tau\right) d s \\
& \leq \eta^{2}\|u\| \int_{0}^{1} G(1, s) \int_{0}^{1} G(1, \tau) d \tau d s \\
& \leq\|u\|
\end{aligned}
$$

Let $\Omega_{1}=\left\{u \in E:\|u\|<\frac{H_{1}}{2}\right\}$

then

$$
\|\mathrm{A} u\| \leq\|u\|, \quad \forall u \in P \cap \partial \Omega_{1}
$$

On the other hand, from $\left(A_{2}\right)$ there exist four positive numbers $M, M^{\prime}, C_{1}$ and $C_{2}$ such that

$$
\begin{aligned}
& f(t, u) \geq M u-C_{1} \quad \forall(t, u) \in[0,1] \times R^{+} \\
& g(t, u) \geq M^{\prime} u-C_{2} \quad \forall(t, u) \in[0,1] \times R^{+}
\end{aligned}
$$

and

$$
M \int_{0}^{1} G(1, s) q(s) d s \geq 2, \quad M^{\prime} \int_{0}^{1} G(1, s) q(s) d s \geq 1 .
$$

By direct computation, for $u \in P$

$$
\begin{aligned}
A u(1) & =\int_{0}^{1} G(1, s) f\left(s, \int_{0}^{1} G(s, \tau) g(\tau, u(\tau)) d \tau\right) d s \\
& \geq \int_{0}^{1} G(1, s)\left[M \int_{0}^{1} G(s, \tau) g(\tau, u(\tau)) d \tau-\mathrm{C}_{1}\right] d s \\
& \geq M \int_{0}^{1} G(1, s) \int_{0}^{1} G(s, \tau)\left[M^{\prime} u(\tau)-C_{2}\right] d \tau d s-C_{1} \int_{0}^{1} G(1, s) d s \\
& \geq M M^{\prime} \int_{0}^{1} G(1, s) q(s) d s \int_{0}^{1} G(1, \tau) d \tau-C_{3}(1)
\end{aligned}
$$

where

$$
\begin{aligned}
C_{3}(1)= & M C_{2} \int_{0}^{1} G(1, s) \int_{0}^{1} G(s, \tau) d \tau d s+C_{1} \int_{0}^{1} G(1, s) d s \\
& \leq M C_{2} \int_{0}^{1} G(1, s) q(s) d s \int_{0}^{1} G(1, \tau) d \tau+C_{1} \int_{0}^{1} G(1, s) d s \\
& =C_{3}
\end{aligned}
$$


Therefore

$$
A u(1) \geq M \int_{0}^{1} G(1, s) q(s) d s M^{\prime} \int_{0}^{1} G(1, \tau) u(\tau) d \tau-C_{3} \geq 2\|u\|-C_{3}
$$

from which if follows that $\|\mathrm{A} u\| \geq A u(1)>\|u\|$, as $\|u\| \rightarrow \infty$

Let $\Omega_{1}=\left\{u \in E:\|u\|<H_{2}\right\}$.Then for $u \in P$ and $\|u\|=H_{2}>0$ sufficient large, we have

$$
\|\mathrm{A} u\| \geq\|u\|, \quad \forall u \in P \cap \partial \Omega_{2}
$$

Thus, from (3.1), (3.2) and Lemma 3, we know that the operator A has a fixed point in $\mathrm{P} \cap\left(\bar{\Omega}_{2} \backslash \Omega_{1}\right)$. The proof is complete.

Theorem 2 If $\left(A_{3}\right)$ and $\left(A_{4}\right)$ are satisfied, then BVP (1.3) has at least one positive solution $(u, v) \in C^{3}\left([0,1], R^{+}\right) \times C^{3}\left([0,1], R^{+}\right)$satisfying $u(t)>0, v(t)>0$.

Proof. From $\left(A_{3}\right)$ there is a number $H_{3} \in(0,1)$, such that for each $(t, u) \in[0,1] \times\left(0, H_{3}\right)$, one has

$$
f(t, u) \geq \lambda u \quad g(t, u) \geq \dot{\lambda}^{\prime} u,
$$

where $\lambda, \dot{\lambda}^{\prime}>0$ satisfies

$$
\lambda \int_{0}^{1} G(1, s) q(s) d s \geq 1 \quad \lambda^{\prime} \int_{0}^{1} G(1, s) d s \geq 1
$$

For every $u \in P$ and $\|u\|=H_{3}$,

$$
\begin{aligned}
A u(1) & =\int_{0}^{1} G(1, s) f\left(s, \int_{0}^{1} G(s, \tau) g(\tau, u(\tau)) d \tau\right) d s \\
& \geq \lambda \int_{0}^{1} G(1, s) \int_{0}^{1} q(s) G(1, \tau) g(\tau, u(\tau)) d \tau d s \\
& \geq \lambda \int_{0}^{1} q(s) G(1, s) \lambda^{\prime} \int_{0}^{1} G(1, \tau) u(\tau) d \tau d s \\
& \geq \lambda\|u\| \int_{0}^{1} q(s) G(1, s) d s \lambda^{\prime} \int_{0}^{1} G(1, \tau) d \tau \\
& \geq\|u\|
\end{aligned}
$$

Let $\Omega_{3}=\left\{u \in E:\|u\|<H_{3}\right\}$, we have

$$
\|\mathrm{A} u\| \geq\|u\|_{,} \quad \forall u \in P \cap \partial \Omega_{3}
$$

On the other hand, we know from $\left(A_{4}\right)$ that there exist three positive numbers $\eta^{\prime}, C_{4}$ and $C_{5}$ such that for every $(t, u) \in[0,1] \times R^{+}$, 


$$
f(t, u) \leq \eta^{\prime} u+C_{4} \quad g(t, u) \leq \eta^{\prime} u+C_{5},
$$

where

$$
\eta^{\prime} \int_{0}^{1} \mathrm{G}(1, \mathrm{~s}) \mathrm{ds} \leq \frac{1}{2}
$$

Thus we have

$$
\begin{aligned}
A u(t) & =\int_{0}^{1} G(t, s) f\left(s, \int_{0}^{1} G(s, \tau) g(\tau, u(\tau)) d \tau\right) d s \\
& \leq \int_{0}^{1} G(1, s) f\left[\eta^{\prime} \int_{0}^{1} G(1, \tau) g(\tau, u(\tau)) d \tau+\mathrm{C}_{4}\right] d s \\
& \leq \eta^{\prime} \int_{0}^{1} G(1, s) d s \int_{0}^{1} G(1, \tau)\left[\eta^{\prime} u(\tau)+C_{5}\right] d \tau+C_{4} \int_{0}^{1} G(1, s) d s \\
& \leq \frac{1}{4}\|u\|+C_{6}
\end{aligned}
$$

where

$$
\mathrm{C}_{6}=\mathrm{C}_{5} \eta^{\prime} \int_{0}^{1} \mathrm{G}(1, \mathrm{~s}) \mathrm{ds} \int_{0}^{1} G(1, \tau) d \tau+C_{4} \int_{0}^{1} G(1, s) d s
$$

from which it follow

$$
\mathrm{A} u \leq\|u\|, a s\|u\| \rightarrow \infty . \text { Let } \Omega_{4}=\left\{u \in E:\|u\|<H_{4}\right\}
$$

For each $u \in P$ and $\|u\|=H_{4}>0$ large enough, we have

$$
\|\mathrm{A} u\| \leq\|u\|, \quad \forall u \in P \cap \partial \Omega_{4}
$$

From (3.3) and (3.4 $\}$ ) and Lemma 3, we know that the operator A has a fixed point in $\mathrm{P} \cap\left(\bar{\Omega}_{2} \backslash \Omega_{1}\right)$. The proof is complete.

Theorem 3 If $\left(A_{2}\right),\left(A_{3}\right)$ and $\left(A_{5}\right)$ are satisfied, then BVP (1.3) has at least two distinct positive solution $\left(u_{1}, v_{1}\right) \quad\left(u_{2} v_{2}\right) \in C^{3}\left([0,1], R^{+}\right) \times C^{3}\left([0,1], R^{+}\right)$satisfying $u_{\mathrm{i}}(t)>0, v_{i}(t)>0,(\mathrm{i}=1,2)$.

Proof. Note that $G(t, s) \leq \frac{1}{2}$. Let $\$_{B_{N}}=\{u \in E:\|u\| \prec \mathrm{N}\}$. Then from $\left(A_{5}\right)$, for every $u \in P \cap \partial B_{N}, t \in[0,1]$, we have

$$
\begin{aligned}
A u(t) & =\int_{0}^{1} G(t, s) f\left(s, \int_{0}^{1} G(s, \tau) g(\tau, u(\tau)) d \tau\right) d s \\
& \leq \frac{1}{2} \int_{0}^{1} f\left(s, \int_{0}^{1} \frac{1}{2} g(\tau, N) d \tau\right) d s \\
& \leq \frac{1}{2} 2 N=N
\end{aligned}
$$


Thus

$$
\|\mathrm{A} u\| \leq\|u\|, \quad \forall u \in P \bigcap \partial B_{N}
$$

And from $\left(A_{2}\right)$ and $\left(A_{3}\right)$ we have

$$
\begin{gathered}
\|\mathrm{A} u\| \geq\|u\|, \quad \forall u \in P \bigcap \partial \Omega_{2} \\
\|\mathrm{~A} u\| \geq\|u\|, \quad \forall u \in P \bigcap \partial \Omega_{3}
\end{gathered}
$$

We can choose $\mathrm{H}_{1}, \mathrm{H}_{2}$ and $\mathrm{N}$ such that $\mathrm{H}_{1} \leq \mathrm{N} \leq \mathrm{H}_{2}$ and (3.5),(3.6),(3.7) are satisfied. From Lemma 4, A has at least two fixed points in $\mathrm{P} \cap\left(\bar{\Omega}_{2} \backslash \overline{\mathrm{B}}_{\mathrm{N}}\right)$ and $\mathrm{P} \cap\left(\mathrm{B}_{\mathrm{N}} \backslash \Omega_{2}\right)$, respectively. The proof is complete.

Examples. Some examples are given to illustrate our main results.

(1) Let $f(t, v)=v^{3}+v^{2}, g(t, u)=u^{3}$, then conditions of Theorem 1 are satisfied. From Theorem 1, BVP (1.3) has at least one positive solution.

(2) Let $f(t, v)=v^{\frac{1}{3}}, g(t, u)=u^{\frac{1}{3}}$, then conditions of Theorem 2 are satisfied. From Theorem 2, BVP (1.3) has at least one positive solution.

(3) Let $f(t, v)=\frac{1}{8}\left(v^{\frac{1}{3}}+v^{3}\right), g(t, u)=2\left(u^{\frac{1}{3}}+u^{3}\right)$. Thus $\mathrm{N}=1$ and

$$
f\left(t, \int_{0}^{1} \frac{1}{2} g(s, N) d s\right)=f(t, 2)=\frac{1}{8}\left(2^{3}+2^{\frac{1}{3}}\right)<2
$$

so conditions of Theorem 3 are satisfied. From Theorem 3, BVP (1.3) has at least two positive solution.

\section{References}

[1] D. R. Anderson, Green's function for a third-order generalized right focal problem, J. Math. Anal. Appl.288 (2003) 1-14.

[2] D. R. Anderson, J. M. Davis, Multiple solutions and eigenvalues for three-order right focal boundary value problems, J. Math. Anal. Appl.267(2002) 135-157.

[3] R. Ma, Multiplicity results for a third order boundary value problem at resonance, Nonliner Anal. TMA 32(4)(1998)493-499

[4] Y. Sun, Positive solutions of a singular third-order three-point boundary value problem, J. Math. Appl.306(2005)589-603.

[5] Q. Yao. The existence and multiplicity of positive solutions for a third-order three-point boundary value problem, Acta Math. Appl 2003, 19: 117-122.

[6] M. E. Shahed, Positive solutions for nonlinear singular third order boundary value problem, Communications in Nonlinear Science and Numerical Simulation. 14(2009): 424-429.

[7] Z. L. Yang, J. X. Sun, Positive solutions of boundary value problems for system of nonlinear second order ordinary dierential equations, Acta Math.Sinica, 47(1)(2004):111-118. 

of Nonlinear Third-Order Differential Equations

[8] R. P. Agarwal, D. O'Regan, P. J. Y. Wong. Positive Solutions of Di erential,Dierence and Integral Equations, Kluwer, Dordrecht, 1999.

[9] Klaus Deiming, Nonlinear Functional Analysis,Springer-Verlag, New York, 1985

[10] Dajun Guo and V. Lakshmikantham, Nonlinear Problems in Abstract Cones, Academic Press, Boston, 1988

[11] M. A. Krasbosel'skii. Positive Solutions of Operator Equations, Noordho, Groningen, 1964. 